\title{
Non-Resonant Background Suppression in Preresonance CARS Spectra of Flavin Adenine Dinucleotide: Demonstration of a Background Suppression Technique using Phase Mismatching and Comparison with the Polarization-Sensitive CARS Technique
}

T. A. H. M. Scholten, ${ }^{*}$ G. W. Lucassen, E. Koelewijn, F. F. M. de Mul and J. Greve

University of Twente, P.O. Box 217, 7500AE Enschede, The Netherlands

\begin{abstract}
Polarization-sensitive CARS spectra of a $5.7 \times 10^{-3} \mathrm{~mol} \mathrm{dm}{ }^{-3}$ flavin adenine dinucleotide (FAD) solution were recorded under preresonance conditions at a pump wavelength of $532 \mathrm{~nm}$. The depolarization ratios of the vibrations are shown to be close to the depolarization ratio of the non-resonant background. This results in a severe reduction of the vibration resonant signal (a factor of 700-900) in the polarization CARS spectrum, and a poor improvement in the ratio of the resonant signal and the non-resonant background $(<10)$.

In this context, a non-resonant background suppression technique is discussed and demonstrated for $5.7 \times 10^{-3}$ and $1.4 \times 10^{-3} \mathrm{~mol} \mathrm{dm}^{-3}$ FAD solutions excited at $532 \mathrm{~nm}$; tite non-resonant susceptibility of the walls of the cuvette, which contains the FAD solution, is used to compensate the non-resonant signal contribution of the solution. An improvement in the signal-to-noise ratio of $\mathrm{ca} .50$ is achieved at the cost of a factor of 30 in the resonant signal strength. Lorentzian-shaped spectral bands are obtained, facilitating the determination of band position, width and intensity. Line shape parameters and depolarization ratios for FAD are extracted from the presented spectra by curve fitting. The signal strength and background suppression achieved with these techniques and the resonance CARS technique (at a pump wavelength of $480 \mathrm{~nm}$ ) are compared and discussed.
\end{abstract}

\section{INTRODUCTION}

CARS (Coherent anti-Stokes Raman scattering) is a non-linear Raman spectroscopic technique which, in the field of biophysics, is especially suitable for the study of fluorescing biological molecules. ${ }^{1}$ A major drawback in CARS spectroscopy, however, is the non-resonant background, which is caused by susceptibility contributions that do not resonate on a vibration transition of the molecule under study. ${ }^{2}$ Especially the pulse-to-pulse fluctuations on this background, caused by the pulsed laser system that is generally used in a CARS experiment, limit the detection of weak vibrational modes of a sample or a dilute solution. ${ }^{3}$

Several methods have been reported for overcoming the problem of the non-resonant background. ${ }^{4-7}$ Among these, the resonance CARS technique ${ }^{5}$ and the polarization-sensitive CARS technique ${ }^{4}$ are the most commonly used. As was shown by Brakel et al., ${ }^{8}$ these techniques may be combined to obtain a superior background suppression.

In this paper, we discuss a background suppression technique that employs phase mismatching in order to compensate coherently the non-resonant signal of the sample using the non-resonant signal of the glass cuvette walls. ${ }^{9,10}$ The technique is demonstrated for the fluorescing biological chromophore flavin adenine dinucleotide (FAD), which is preresonantly excited at a pump wavelength of $532 \mathrm{~nm}$.

Polarization-sensitive CARS spectra of FAD, recorded under the same preresonance conditions, are presented. The depolarization ratios of the preresonantly enhanced vibrations are shown to be close to $1 / 3$, i.e. the depolarization ratio of the non-resonant background. As a result, the shape of the spectrum changes rapidly when recording the CARS signal under slightly different polarization orientations. This permits an accurate determination of the depolarization ratios of the vibrations. Further, the vibration resonant signal is shown to be strongly reduced on suppressing the nonresonant background.

This case of nearly equal depolarization ratios of vibration resonant signal and non-resonant background, which in general can occur when a molecule is (pre)resonantly excited, ${ }^{11}$ favours the background suppression technique by phase mismatching: the signal level is 50-100 times higher than the intensity of the polarization CARS spectra, and good spectra of lower concentrations of FAD could be recorded.

A comparison is made concerning the signal strength, the quality and differences of the spectra of FAD, obtained with the phase-mismatching technique, the polarization CARS technique and the resonance CARS 
technique. Spectra of FAD obtained with the resonance CARS method were previously published by Dutta $e t$ $a l .{ }^{12}$

\section{PRINCIPLE OF THE BACKGROUND SUPPRESSION TECHNIQUE BY PHASE MISMATCHING}

The non-resonant background suppression technique by phase mismatching uses a sample geometry that is usually encountered in a CARS experiment. The sample is enclosed between two cell windows (cuvette walls) and thus forms a triple layer as shown schematically in Fig. 1. The non-resonant susceptibility of these cells windows is a problem in CARS spectroscopy as it generates part of the unwanted non-resonant background. Its effect can be made small by using a sample layer thickness in excess of the thickness of the cell walls. In case of absorbing [i.e. (pre)resonantly excited] media, this usually cannot be achieved since the sample thickness is often limited to less than $1 \mathrm{~mm}$ owing to absorption of the pump and/or Stokes laser beams and the CARS signal. However, it is possible to take advantage of the non-resonant contribution of the cuvette windows by using it to compensate the non-resonant contribution originating from the sample layer.

As is well known, ${ }^{13}$ the CARS signal intensity in the plane wave approximation is proportional to the following parameters:

$$
I_{\text {CARS }} \propto\left|\chi^{(3)}\right|^{2} l^{2} \operatorname{sinc}^{2}(\Delta k l / 2)
$$

where $\chi^{(3)}$ is the third-order susceptibility, $l$ the sample length or length of the overlap region of the laser beams and $\Delta k$ the phase mismatch of the wave vectors of the pump ( $\left.\mathbf{k}_{\mathrm{p}}\right)$, Stokes $\left(\mathbf{k}_{\mathrm{s}}\right)$ and CARS ( $\mathbf{k}_{\text {CARS }}$ ) beams. The phase mismatch, $\Delta k$, depends on the crossing angle $\theta$ of the pump and Stokes beams in the sample: for a single degenerate CARS process,

$$
\Delta k=\left|\mathbf{k}_{\mathrm{CARS}}\right|-\left[4\left|\mathbf{k}_{\mathbf{p}}\right|^{2}+\left|\mathbf{k}_{\mathrm{s}}\right|^{2}-4\left|\mathbf{k}_{\mathrm{p}} \| \mathbf{k}_{\mathrm{s}}\right| \cos (\theta)\right]^{1 / 2}
$$

The signal intensity varies sinusoidally with $\Delta k l$, and a so-called coherence length, $L_{\mathrm{COH}}$, of the sample can be defined for which the signal reaches a maximum:

$$
L_{\mathrm{COH}}=\pi / \Delta k
$$

Regarding the triple layer (Fig. 1), two different values of $l$ can be distinguished: for the non-resonant suscepti-
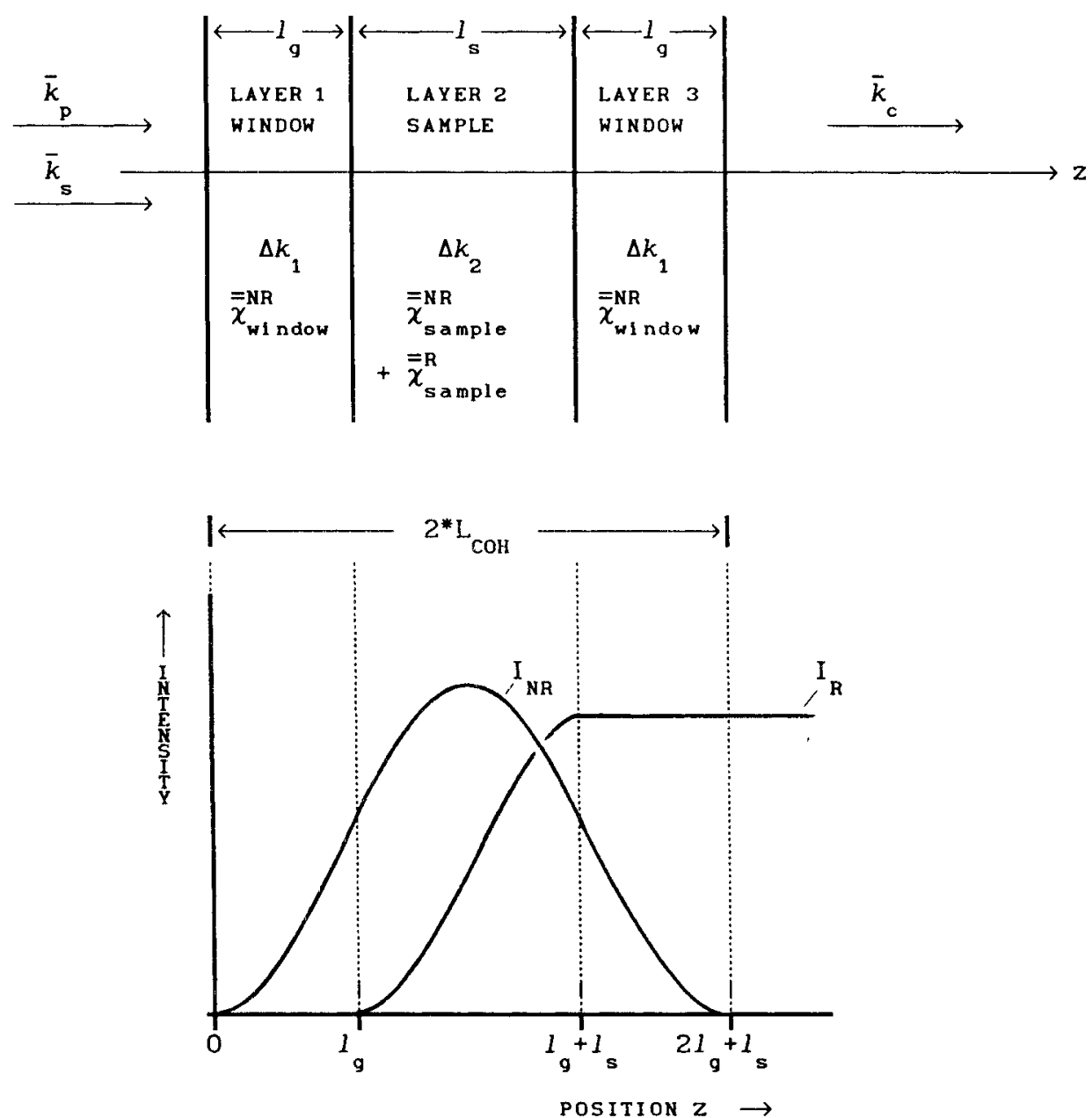

Figure 1. Schematic triple layer configuration consisting of two window layers $/ g$, enclosing the sample layer $/ \mathrm{s}$. The window layers only contribute to the non-resonant CARS signal. By appropriate selection of the phase mismatches $\Delta k_{1}$ and $\Delta k_{2}$, the total triple layer thickness $\left(2 I_{\mathrm{g}}+I_{\mathrm{s}}\right)$ equals $2 L_{\mathrm{COH}}$ and the non-resonant contribution cancels (indicated schematically in the lower figure). Since $I_{\mathrm{s}}<2 L_{\mathrm{coH}}$ the vibration resonant contribution remains. (A detailed discussion is given in the Appendix.) 
bility, the total triple layer thickness $2 l_{\mathrm{g}}+l_{\mathrm{s}}$ applies to $l$, whereas for the vibration resonant susceptibility the sample layer thickness $l_{\mathrm{s}}$ applies to $l$. When selecting a crossing angle $\theta$ and thus a phase-mismatch $\Delta k$, so that $L_{\mathrm{COH}}$ is half the total triple layer thickness, $2 l_{\mathrm{g}}+l_{\mathrm{s}}$, the $\operatorname{sinc}^{2}(\Delta k l / 2)$ function is zero and the non-resonant contribution vanishes. This condition is illustrated in Fig. 1. The sample layer thickness $l_{\mathrm{s}}$ is smaller than $2 L_{\mathrm{COH}}$ and a resonant contribution therefore remains present.

In case the sample thickness $l_{\mathrm{s}}$ equals $L_{\mathrm{COH}}$, the vibration resonant signal loss is $(\pi / 2)^{2}=2.5$, compared with the case of the same sample layer under matching conditions $\left(L_{\mathrm{COH}}=\infty\right)$.

In general, the dispersion and non-resonant susceptibility of the cell windows and the sample layer will differ and the qualitative picture in Fig. 1 becomes more complex. In the case of a transparent sample layer it was shown, ${ }^{9}$ theoretically and experimentally, that an angle $\theta$ can be found for which the non-resonant background is minimized. The reported background suppression in the spectrum of ethanol was best for a thin sample layer; in case of a $0.25 \mathrm{~mm}$ ethanol layer between two $0.15-\mathrm{mm}$ microscope cover-glass slips, a factor of ca 50 improvement in resonant signal/ background ratio was demonstrated. For transparent media this figure cannot compete with the improvement that can be obtained by the polarization CARS technique (although the phase-mismatching technique shows other interesting possibilities). Further, the polarization CARS method can be applied to larger sample lengths, which usually more than compensates its greater vibration resonant signal loss $\left[>16\left(1-3 \rho^{\mathbf{R}}\right)^{-2}\right.$, where $\rho^{\mathrm{R}}$ is the depolarization ratio of the vibration $\left.{ }^{13}\right]$.

As will be demonstrated, the non-resonant background suppression technique by phase mismatching seems to be more applicable to the case of absorbing media for two reasons: (i) as stated before, absorbing media usually have an optimum sample thickness which is in the range of $1 \mathrm{~mm}$ and below: for these thicknesses the background suppression technique by phase mismatching is at its best; and (ii) on approaching resonance conditions, the depolarization ratio of many totally symmetric vibrations, which are coupled to the excited electronic transition lying in a definite molecular direction, tend to become equal to $1 / 3 .^{11}$ If the so-called Kleinman symmetry applies, ${ }^{13}$ this is equal to the depolarization ratio of the non-resonant (solvent) background. Then, on suppressing the non-resonant background by the polarization CARS technique, the resonant signal is also suppressed, resulting in severe signal loss. Instead, the mismatching method does not depend on the difference in depolarization ratio of background and resonant signal, while its signal loss (theoretically near a factor of 2.5) remains acceptable.

For the case presented here of an absorbing sample layer, the theoretical intensity of the non-resonant CARS signal of the triple layer structure is derived and discussed in the Appendix. The general formula, Eqn (A8), applies to the preresonantly excited spectra of the FAD solutions. For the triple layer used for the phasemismatched measurements, the glass to sample thickness ratio is $l_{\mathrm{g}} / l_{\mathrm{s}}=0.6$. The ratio of the (real) nonresonant susceptibility of an aqueous solution with respect to the non-resonant susceptibility of the glass windows was measured to be $F_{\mathrm{r}}=0.88$. Hence the middle plot in Fig. A1 approximately applies to the FAD measurements (assuming $\Delta k_{1} / \Delta k_{2} \approx 1$ ).

\section{EXPERIMENTAL}

The experimental CARS spectrometer (described in more detail elsewhere ${ }^{14}$ ) consists of a frequency doubled Nd:YAG laser (Quanta-Ray DCR2), which provides part of the 532-nm pump beam, and a pulsed dye laser (Quanta-Ray PDL2), which is operated in the 800-1650 $\mathrm{cm}^{-1}$ range, using Rhodamine $6 \mathrm{G}$. For the polarization CARS measurements, the pump and Stokes beams are linearly polarized, and the signal polarization is analysed using Glan-Taylor polarizers. The beams are focused and refracted under and the desired crossing angle using a $250-\mathrm{mm}$ focal length lens. The spot size at the sample position is ca $100 \mu \mathrm{m}$. The signal is spatially filtered with an iris diaphragm and spectrally filtered using two coupled $0.2-\mathrm{m}$ monochromators (JY H20-UV), operated in the additive mode. Detection is performed with a photomultiplier tube. For ease of operation, the setup is largely computer controlled.

FAD was a gift from A. J. W. G. Visser of the Agricultural University (Wageningen, The Netherlands). The material is dissolved in distilled water and the concentration is determined from absorption spectra (recorded with a Beckman UV-Vis DU-8 spectrophotometer), using the known molar absorptivities of FAD (11300 $1 \mathrm{~mol}^{-1} \mathrm{~cm}^{-1}$ at $450 \mathrm{~nm}$ and $93001 \mathrm{~mol}^{-1} \mathrm{~cm}^{-1}$ at 375 $\mathrm{nm}^{15}$ ). The sample solutions are contained in a cuvette with standard microscope cover-glass slips as cell windows (thickness $l_{\mathrm{g}}=0.15 \mathrm{~mm}$ ). The cover-glasses are anti-reflection coated on their air/glass interface to avoid multiple interference effects, previously reported. ${ }^{14}$ The sample pathlength, $l_{s}$, is determined by an exchangeable spacer: $0.9 \mathrm{~mm}$ for the polarization CARS measurements and $0.25 \mathrm{~mm}$ for the measurements using phase mismatching.

Phase matching for the normal CARS and polarization CARS measurements is provided by automatically tuning the matching and signal collection angle (Fig. 2) via a predetermined linear function.

For phase-mismatched measurements the scanning procedure is basically the same: the beam crossing angle and signal collection angle are adjusted according

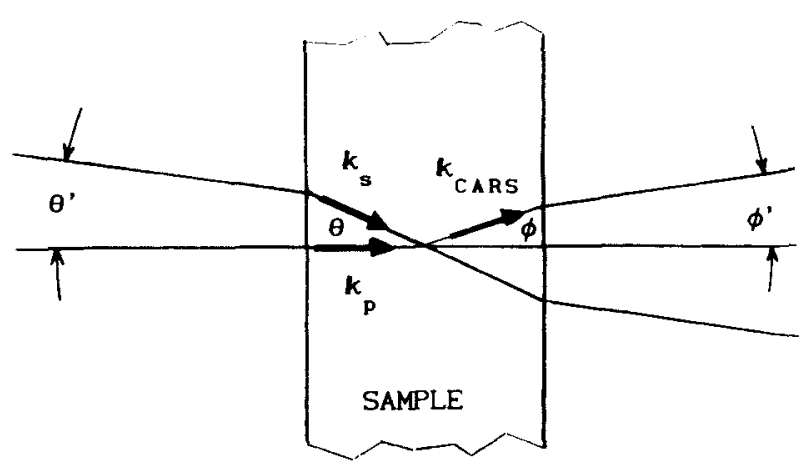

Figure 2. Beam crossing and signal collection geometry: $\theta$ internal, $\theta^{\prime}$ external beam crossing angle; $\phi$ internal, $\phi^{\prime}$ external signal collection angle. If the vector sum $2 \mathbf{k}_{\mathrm{p}}-\mathbf{k}_{\mathrm{s}}-\mathbf{k}_{\text {caps }}$ equals zero, the crossing angle $\theta$ is the so-called matching angle. 
to a predetermined function. However, the beam crossing angle, $\theta$, is tuned from the matching angle towards a higher value. At this angle the phase mismatches in the cell windows and the sample layer are such that the total thickness concerning the non-resonant contribution, $2 l_{\mathrm{g}}+l_{\mathrm{s}}$, is twice the coherence length and a minimum therefore occurs in the non-resonant background. The signal collection angle is then adjusted for maximum resonant-to-non-resonant signal and a Lorentzian shape of the spectral bands. For the $0.15 /$ $0.25 / 0.15 \mathrm{~mm}$ glass/FAD solution/glass triple layer, the beam crossing angle $\theta^{\prime}$ and collection angle $\phi^{\prime}$ (externally measured values) are scanned according to $\theta^{\prime}=1.89+5.44 \times 10^{-4} \sigma$ (degrees) and $\phi^{\prime}=-1.70$ $-2.90 \times 10^{-4} \sigma$ (degrees), where $\sigma$ is the Raman shift in $\mathrm{cm}^{-1}$.

No polarizers are used for the mismatched measurements; in that case the polarizations of the pump and
Stokes laser are nearly linear (>95\%), and oriented parallel to each other.

All CARS spectra shown are scans (or added scans) of 300 measurement points with 25 pulses averaged per point, and are smoothed with a five-point SavitzkyGolay procedure. For signal comparison, the dependence of the sensitivity of the detection system on the polarization orientation of the CARS signal is accounted for.

\section{ABSORPTION SPECTRUM AND EXCITATION CONDITIONS FOR FAD}

Excitation of FAD with a pump wavelength of $532 \mathrm{~nm}$ is unique in that the pump and Stokes beams are hardly absorbed by the sample. Figure 3 shows the molecular
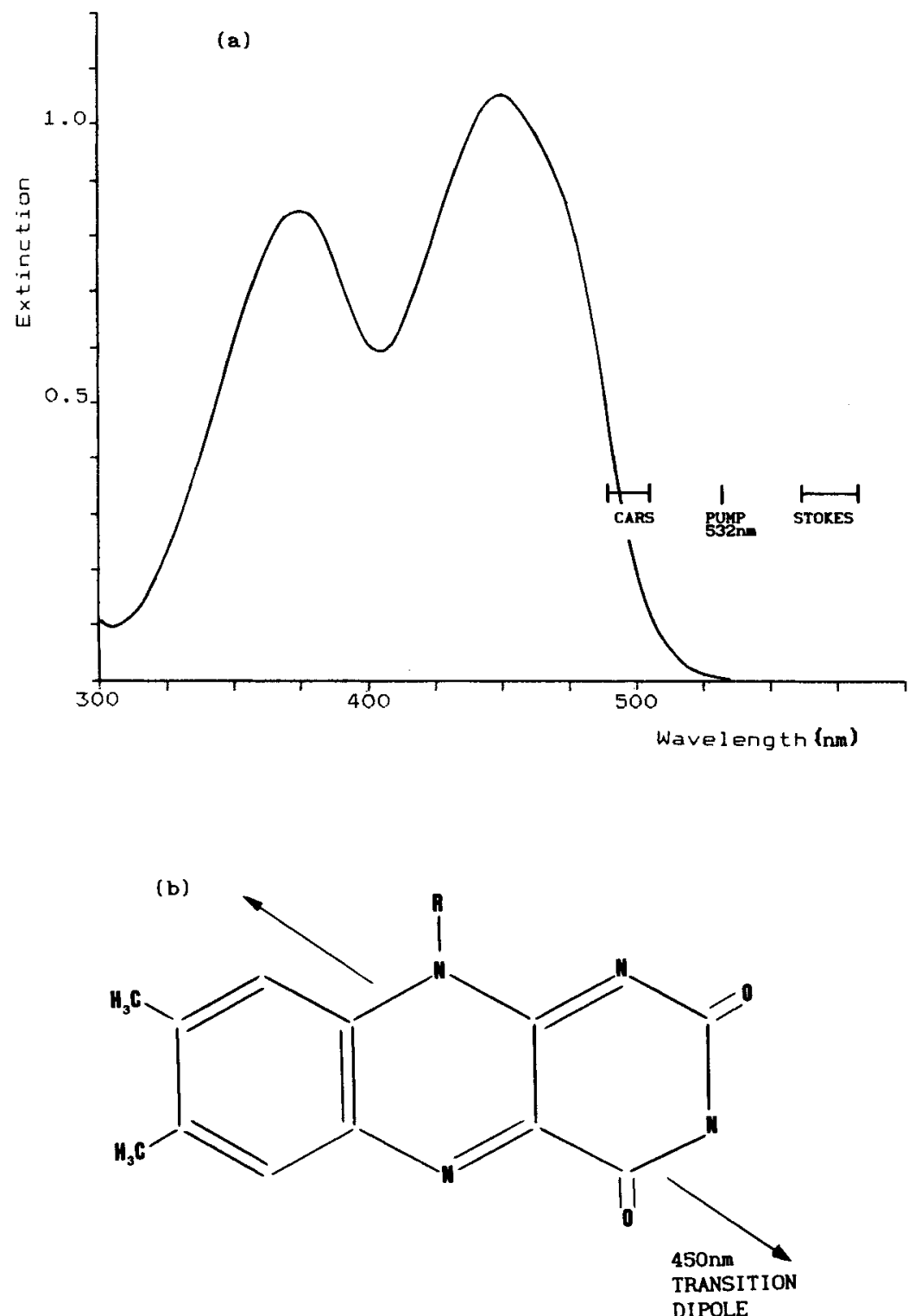

Figure 3. (a) Absorption spectrum of a ca. $10^{-4} \mathrm{~mol} \mathrm{dm}-3$ FAD solution in a 1 -cm pathlength cell. Indicated are the positions of the $532-\mathrm{nm}$ pump wavelength and the $1000-1650 \mathrm{~cm}^{-1}$ Stokes and anti-Stokes range, which is the Raman window used throughout this paper. (b) Molecular structure of the flavin chromophore of FAD and orientation of the electric transition dipole moment associated with the 450- $\mathrm{nm}$ absorption band. ${ }^{12,16} \mathrm{R}$ indicates a ribose-phosphate-adenosine group attached to the chromophore. 
structure of the chromophore of FAD and the absorption spectrum of a ca. $10^{-4} \mathrm{~mol} \mathrm{dm}{ }^{-3}$ FAD solution in a 1-cm pathlength cell. Also indicated are the pump wavelength and the wavenumber range of the Stokes and CARS signal $\left(1000-1650 \mathrm{~cm}^{-1}\right)$ used in our experiments.

The sample is seen to be preresonantly excited by the CARS signal only, while absorption at the pump and Stokes wavelengths is negligible. According to excitation profiles determined by resonance Raman spectroscopy, ${ }^{16}$ maximum signal enhancement is obtained around $480-490 \mathrm{~nm}$, which is near a $\pi-\pi^{*}$ transition. On tuning the CARS signal from 1000 to $1650 \mathrm{~cm}^{-1}$ (relative to a pump wavelength of $532 \mathrm{~nm}$ ), its wavelength changes from 505 to $489 \mathrm{~nm}$ and comes closer to the 480-490 $\mathrm{nm}$ electronic transition, yielding an increased pre-resonance enhancement.

This excitation situation is different from the usually encountered excitation scheme at a pump wavelength of $480 \mathrm{~nm},{ }^{12,17}$ at which the vibrational bands of flavins appear as approximate positive Lorentzian curves in the resonance CARS spectrum, ${ }^{18}$ and both the pump and CARS wavelengths are in resonance with the FAD molecule.

The decrease in signal strength due to preresonance excitation at $532 \mathrm{~nm}$, compared with resonant excitation at $480 \mathrm{~nm}$, can be compensated since the molecule can withstand higher power levels at the pump and Stokes wavelengths before degrading. In fact, no degradation was observed at the power levels used to obtain the spectra presented in this paper. The absence of a rigorous resonance condition, however, demands higher FAD concentrations and/or a non-resonant background reduction technique.

\section{POLARIZATION CARS SPECTRA}

Figure 4 shows polarization CARS spectra of a $5.7 \times 10^{-3} \mathrm{~mol} \mathrm{dm}^{-3}$ FAD solution in a $0.9-\mathrm{mm}$ pathlength cell. The polarization orientation of the pump beam is rotated over $\alpha=60.0 \pm 0.2^{\circ}$ with respect to that of the Stokes beam. The polarization orientation of the analyser for minimum non-resonant background is $\beta=58.7 \pm 0.2^{\circ}$ with respect to the polarization orientation of the Stokes beam (see inset, Fig. 4). This corresponds to a non-resonant background depolarization ratio of $\rho^{\mathrm{NR}}=1 /(3.16 \pm 0.03)$.

The reduction of the non-resonant background achieved when the analyser is rotated from $\beta=-\alpha$ (parallel to the polarization orientation of the pump) to $\beta=58.7^{\circ}$ (perpendicular to the polarization orientation of the background) is $4 \times 10^{3}-8 \times 10^{3}$. The improvement in the ratio of the vibration resonant signal and the non-resonant background depends on the depolarization ratio, $\rho^{\mathbf{R}}$, of the vibration and is a factor of $4\left(1-\rho^{\mathrm{R}} / \rho^{\mathrm{NR}}\right)^{-2}$ lower than the background suppression figure (i.e. at least a factor 4 lower for a fully polarized vibration).

In Fig. 4 , the analysing angle $\beta$ is varied by $\Delta \beta=1.26,0.63,0$ and $-0.63^{\circ}$ away from $58.7^{\circ}$. In this way, vibrations which are weak or have a depolar- ization ratio close to $\rho^{\mathrm{NR}}$ can be detected more easily. The rate and sign with which a (dispersive) vibrational contribution varies with $\Delta \beta$ qualitatively indicates the magnitude and sign of $\rho^{\mathrm{R}}-\rho^{\mathrm{NR}}$.

From these spectra, 15 bands are discernible in the $1000-1650 \mathrm{~cm}^{-1}$ range, including the 13 flavin Raman bands (often denoted I-XIII) in the $1100-1700 \mathrm{~cm}^{-1}$ range. The origin of the broad band which extends from 1000 to $1200 \mathrm{~cm}^{-1}$ (and shown to be reproducible) is not clear; as can be seen from Fig. 4, it is polarization sensitive and contains two weak vibrations at 1067 and $1135 \mathrm{~cm}^{-1}$

Using the magnitude (relative to the $1347 \mathrm{~cm}^{-1}$ susceptibility), the wavenumber position and the linewidth of the vibration resonant susceptibilities, which will be determined in the next section, the depolarization ratios were calculated by simulating the polarization CARS spectra using the intensity equations derived by Brakel et al. ${ }^{8}$ Especially the band shape, intensity and rate of change with $\Delta \beta$ were used as criteria for the fit. The broad band at $1000-1200 \mathrm{~cm}^{-1}$ and the apparently remaining non-resonant background in the optimum position of the analyser $\left(\beta=58.7^{\circ}\right)$ were not accounted for. Figure 5 shows the simulated spectra using the line parameters summarized in Table 1.

The non-resonant background was assumed to be real and constant throughout the spectrum. Comparison of the calculated and measured FAD spectrum for $\beta=-\alpha$ [pump, Stokes and CARS field vectors parallel, hence the spectrum proportional to $\left.\left(\chi_{111}\right)^{2}\right]$, showed that the non-resonant susceptibility was approximately 0.7 times the resonant susceptibility of the $1347 \mathrm{~cm}^{-1}$ vibration. A departure from the assumption of a real and constant background may occur for the nonresonant susceptibility of the FAD molecule since it is preresonantly excited; this, however, was not investigated further.

Table 1. Line shape parameters and depolarization
ratios of FAD, determined by curve fitting
the spectra in Figs 8(a) and Fig. 4, respec-
tively

a The normalized depolarization ratio $\rho^{\mathrm{R}} / \rho^{\mathrm{NA}}$ (with $\rho^{N R}=1 / 3.16$ ) is given, since this value determines the polarization CARS line shape. 


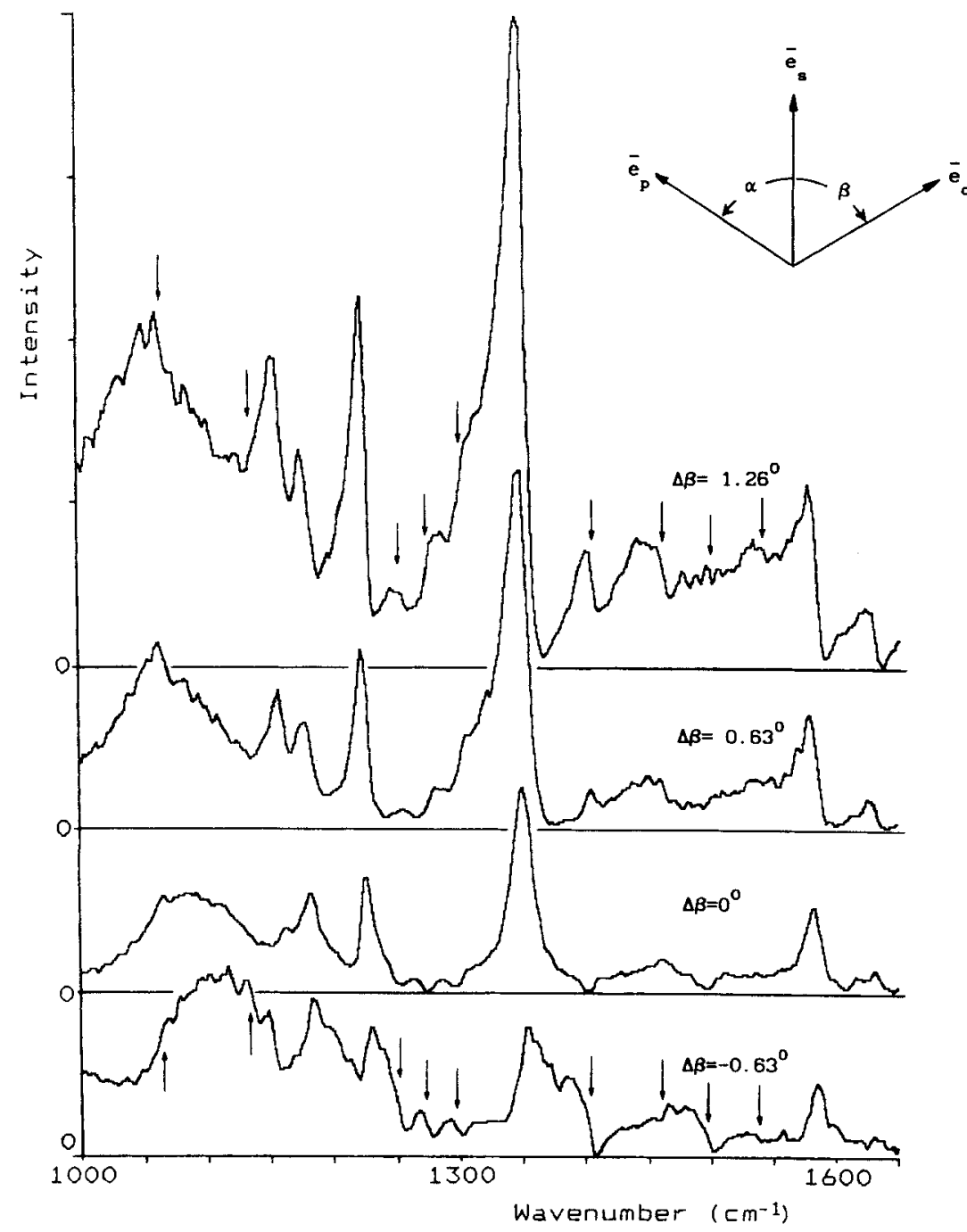

Figure 4. Polarization-sensitive CARS spectra (sum of two or three scans) of a $5.7 \times 10^{-3} \mathrm{~mol} \mathrm{dm}-3$ FAD solution in a $0.9-\mathrm{mm}$ pathlength cell. The spectra are drawn to the same scale; the zero-intensity baseline is shifted and indicated for each spectrum. The polarization orientation of the pump, Stokes and CARS beam $\left(\theta_{p}, \theta_{\mathrm{s}}\right.$ and $\theta_{\mathrm{c}}$, respectively) is depicted in the upper-right corner: $\alpha=60.0^{\circ}, \beta=58.7^{\circ}$. From top to bottom the polarization analyser angle $\beta$ is varied by $1.26,0.63,0$ and $-0.63^{\circ}$, respectively. The weakest vibrations are indicated by an arrow.

For these polarization-sensitive CARS measurements, the pump and Stokes energies incident on the sample are fairly high, ca. 430 and ca. $180 \mu \mathrm{J}$ per pulse, respectively, whereas the CARS signal strength is marginal (i.e. the photomultiplier has to be operated at maximum voltage). This is caused by two effects: firstly, the FAD molecule is only preresonantly enhanced at the antiStokes wavelength, and secondly, the depolarization ratios of the preresonantly enhanced vibrations are nearly equal to $\rho^{\mathrm{NR}}$. This can be concluded from the sensitivity of the polarization CARS spectra for $\Delta \beta$; also, Fig. 6 shows the $\left(\chi_{1111}\right)^{2}$ and $\left(\chi_{1221}\right)^{2}$ spectra $\left(\alpha=45^{\circ}\right.$, and $\beta=-45^{\circ}$ and $45^{\circ}$ respectively) of a 0.25 -mm pathlength FAD solution of the same concentration as used for the polarization CARS measurements. Only minor differences in shape are observed, which can be attributed to pulse-to-pulse variations in the spectra. The vertical scale of the $\left(\chi_{1221}\right)^{2}$ spectrum is enlarged by a factor ca. 11 with respect to the $\left(\chi_{1111}\right)^{2}$ spectrum, indicating a non-resonant depolarization ratio of $1 / 3.3$. The discrepancy with the previously determined figure $(1 / 3.16)$ is probably caused by a difference in the transmission of the detection system for $\beta=45^{\circ}$ and $-45^{\circ}$ polarization.

The decrease in resonant intensity which occurs when the analyser is rotated from $\beta=-\alpha$ to $\beta=58.7^{\circ}$ was measured to be ca. 900 for the $1347 \mathrm{~cm}^{-1}$ vibration (700 calculated from $\rho^{\mathbf{R}}$ in Table 1) and ca. 700 for the 1225 $\mathrm{cm}^{-1}$ vibration (500 calculated), and even higher for most of the other vibrations. The improvement in the signal-to-background ratio (i.e. background suppression factor divided by signal loss) is effectively reduced to less than 10-fold. In this case the (pre)resonantly excited FAD solutions and in general for spectra with depolarization ratios close to $1 / 3$, the polarization CARS technique is (except for obtaining information on depolarization ratios) less useful as a method to suppress the non-resonant background. Especially when 


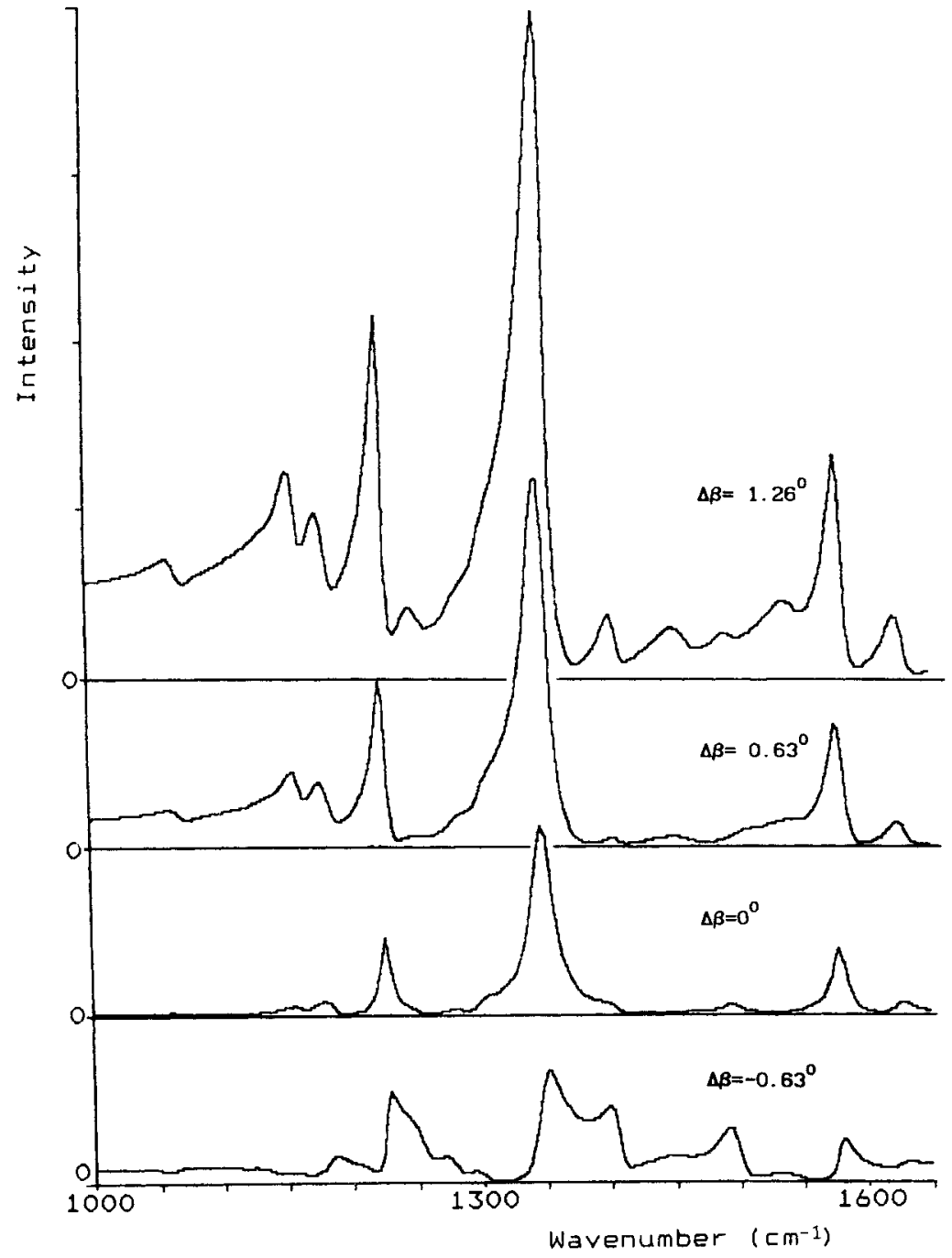

Figure 5. Calculated polarization-sensitive CARS spectra, using the line parameters and depolarization ratios summarized in Table 1. The spectra are drawn to the same scale; the zero-intensity baseline is shifted and indicated for each spectrum. The polarization conditions of the spectra correspond to those in Fig. 4 . The non-resonant susceptibility $x^{\mathrm{NR}}$ is 0.7 times the magnitude of the resonant susceptibility of the $1347 \mathrm{~cm}^{-1}$ vibration.

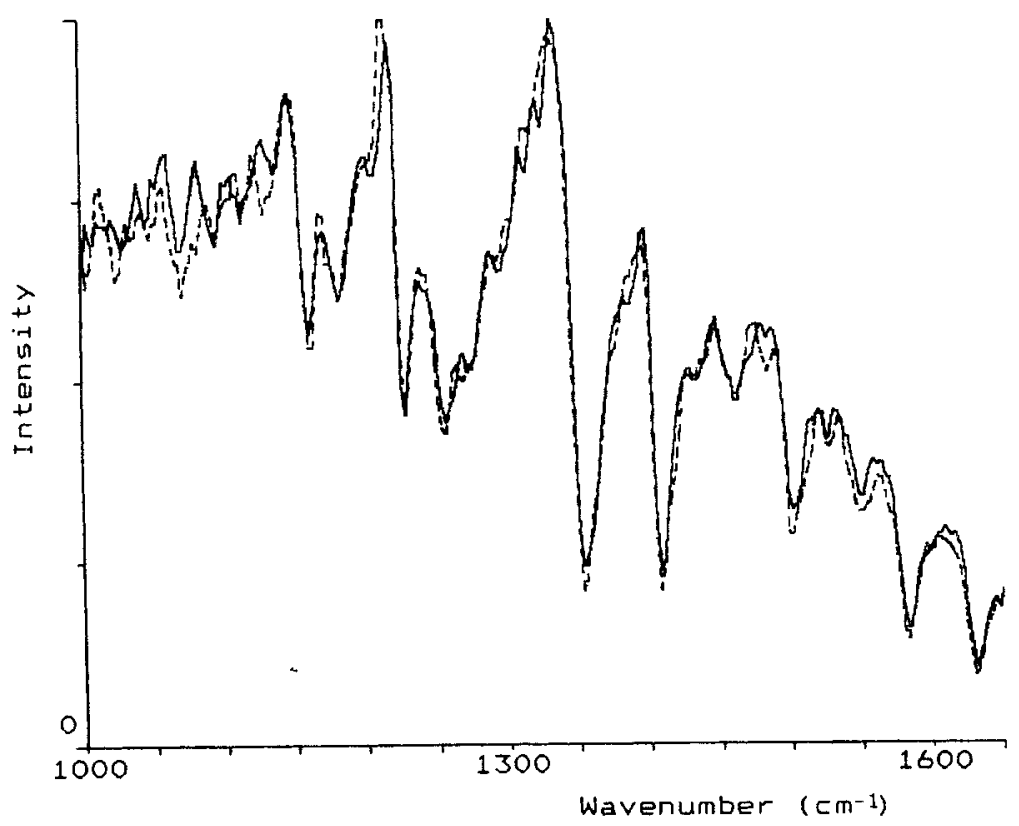

Figure 6. $\left(x_{111}\right)^{2}$ spectrum (solid line) and $\left(x_{1221}\right)^{2}$ spectrum (dashed line) of a $5.7 \times 10^{-3} \mathrm{~mol} \mathrm{dm}^{-3}$ FAD solution in a $0.25-\mathrm{mm}$ pathlength cell with $0.15 \mathrm{~mm}$ cover-glass windows. The scale for the $\left(X_{1221}\right)^{2}$ spectrum is enlarged by a factor of ca. 11 with respect to that of the $\left(x_{1111}\right)^{2}$ spectrum. 


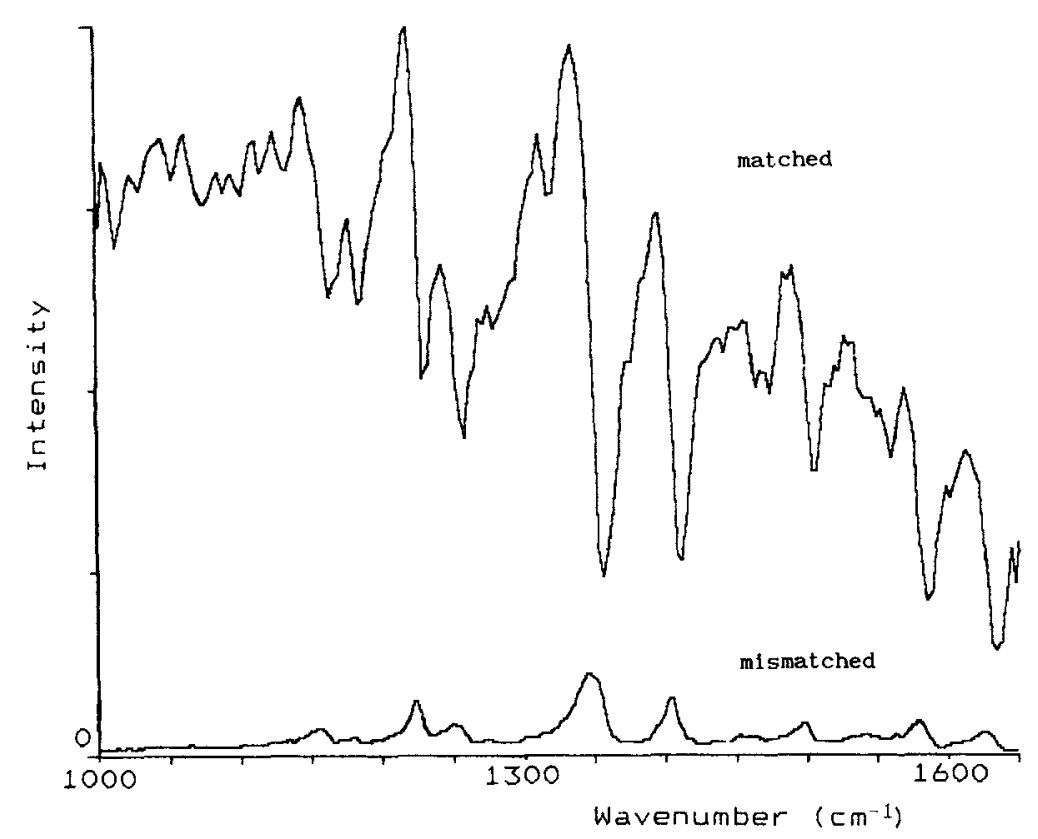

Figure 7. CARS spectra of a $5.7 \times 10^{-3} \mathrm{~mol} \mathrm{dm} \mathrm{m}^{-3} \mathrm{FAD}$ solution in a $0.15 / 0.25 / 0.15 \mathrm{~mm}$ glass/sample/glass configuration. The upper curve is the normal CARS spectrum measured under phase-matching conditions; the lower spectrum is that obtained using the mismatching technique. Both spectra are drawn to the same scale and have the same zero intensity axis. The signal collection aperture is not limited by the iris diaphragm (see text).

lowering the FAD concentration to $1 \times 10^{-3}$ or $2 \times 10^{-3} \mathrm{~mol} \mathrm{dm}^{-3}$ the recording of polarization CARS spectra with reasonable quality was very difficult.

\section{THE PHASE-MISMATChING METHOD}

In the case of FAD excited at $532 \mathrm{~nm}$, the absorption coefficients at the pump and Stokes wavelengths $\left(\alpha_{p}\right.$ and $\alpha_{s}$, respectively), are nearly zero and absorption occurs mainly at the CARS wavelength (determined by the coefficient $\alpha_{c}$ ). For a $0.25-\mathrm{mm}$ pathlength and a $5.7 \times 10^{-3} \mathrm{~mol} \mathrm{dm}^{-3}$ concentration, $\exp \left(-\Delta \alpha l_{\mathrm{s}}\right) \approx$ $\exp \left(\alpha_{\mathrm{c}} l_{\mathrm{s}} / 2\right)$ ranges from 1.2 to 2.1 for a Raman shift of $1000-1650 \mathrm{~cm}^{-1}$ (with $\Delta \alpha=\frac{1}{2}\left(2 \alpha_{\mathrm{p}}+\alpha_{\mathrm{s}}-\alpha_{\mathrm{c}}\right) ;$ see Appendix).

Figure 7 compares, on the same scale, the CARS spectrum of a $0.15 / 0.25 / 0.15 \mathrm{~mm}$ glass/FAD solution/ glass configuration in the case of phase matching and in the case when the beam crossing and signal collection angle are set for maximum suppression of the nonresonant background. Using line shape analysis [i.e. the peak resonant contribution follows from the extremes $I_{\max }$ and $I_{\min }$ of a dispersive line according to $I^{\mathrm{R}}=$ $\left.\left(\sqrt{I}_{\max }-\sqrt{I_{\min }}\right)^{2}\right]$, the reduction in resonant signal is seen to be very near to the above-mentioned factor $(\pi / 2)^{2}$.

A further improvement in the resonant signal-tobackground ratio of the mismatched spectrum can be achieved by limiting the collection angle aperture. This aperture is determined by the iris diaphragm, which is used for spatial filtering the CARS signal. Normally (i.e. for the polarization CARS measurements), the diameter of the diaphragm is larger than the CARS beam diameter and the signal passes unobstructed. In the suppression technique by phase mismatching a minimum in the non-resonant signal only occurs in a sharply defined (signal collection) direction. Apart from this direction, there remains a substantial non-resonant signal as a result of incomplete destructive interference of contributions originating from different parts of the medium. It is caused by the finite dimensions of the polarized part of the medium (finite in the direction perpendicular to the propagation direction of the interacting beams), for which the plane wave approximation, as utilized in the Appendix, is not valid. ${ }^{9}$ If the beam crossing angle is set for a minimum non-resonant background, this effect can be seen by reducing the collection angle aperture and scanning the collection angle: the detected CARS signal will show two maxima with a minimum in between. Scanning is performed with the reduced collection angle aperture retained in this minimum.

The spectrum in Fig. 8(a) for a $5.7 \times 10^{-3} \mathrm{~mol} \mathrm{dm}^{-3}$ FAD concentration was recorded with the iris diaphragm set to $1 \mathrm{~mm}$ diameter (at a distance of $40 \mathrm{~cm}$ from the sample the resolution is $0.14^{\circ}$ ). The improvement in the resonant signal-to-background ratio, compared with the mismatched scan of Fig. 7, is a factor of 2 , at the cost of an extra factor of 6 in the resonant signal intensity due to the reduced collection aperture.

The spectrum with this background suppression technique has peaks with a Lorentzian character which are offset by a remaining non-resonant background. It can be shown ${ }^{9}$ that the dispersive interference between the non-resonant background and vibration resonant contributions is opposed in the above-mentioned maxima and cancel for the collection angle positioned in the minimum. As a result, the background in the spectrum apparently does not interfere with the vibration resonant signal. The determination of the position of the vibrational wavenumbers, the $\left(\chi_{1111}\right)^{2}$ magnitude and the HWHM $\Gamma$ of the vibrations is, however, not directly possible; in addition to Lorentzian contributions, the 


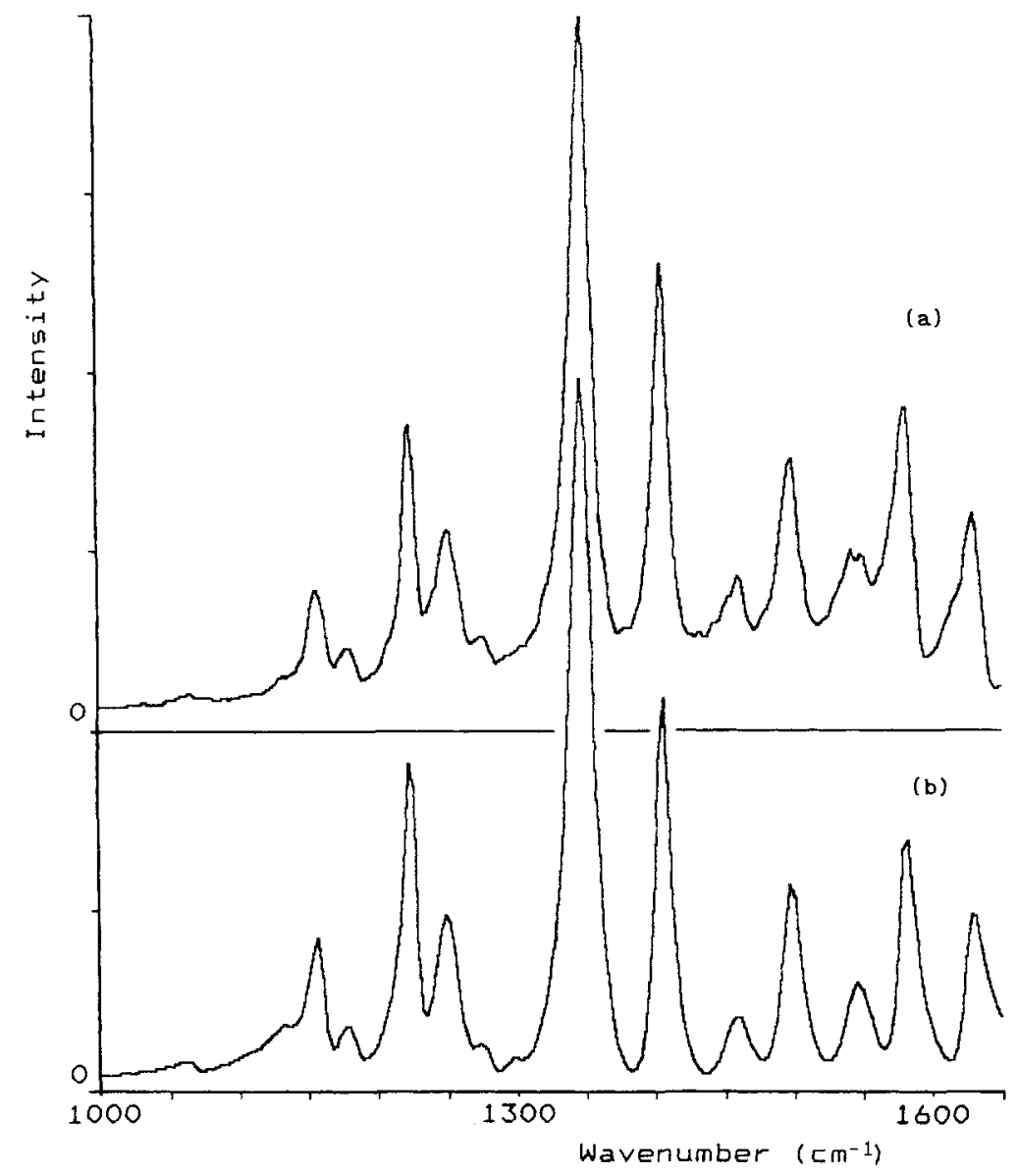

Figure 8. (a) Measured CARS spectrum (sum of five scans) of a $5.7 \times 10^{-3} \mathrm{~mol} \mathrm{dm}^{-3}$ FAD solution in a $0.15 / 0.25 / 0.15 \mathrm{~mm}$ triple layer configuration, using the mismatching technique. For maximum background suppression the spectrum was recorded with a reduced signal collection aperture (resolution $0.14^{\circ}$ ). (b) Calculated CARS spectrum using the line shape parameters summarized in Table 1, and assuming a zero non-resonant contribution.

spectrum contains interference terms between the resonant susceptibilities of neighbouring spectral bands. ${ }^{8,19}$

Starting off with the peak height, width and position of the spectral bands, determined directly from Fig. 8(a), the spectrum, including interference terms, was calculated. The line parameters were then adjusted in a recursive way until a satisfactory fit with both Fig. 8(a) and Fig. 6 was obtained. In the latter situation the triple layer is matched and a non-resonant susceptibility of 1.22 with respect to the susceptibility of the $1347 \mathrm{~cm}^{-1}$ vibration gave the best fit.

It was found that the interference term mainly affects the peak heights of the weaker vibrations (up to $100 \%$ ). The position of the vibrations had to be adjusted by less than $3 \mathrm{~cm}^{-1}$ and the width of a few spectral bands had to be adjusted by $10-20 \%$.

Figure $8(\mathrm{~b})$ gives the calculated CARS spectrum, using the fitted line parameters which are summarized in Table 1, and assuming that the non-resonant susceptibility is zero. Major deviations from the measured spectrum occur at the low and high wavenumber edges; owing to the absence of vibrations outside the 1000 $1650 \mathrm{~cm}^{-1}$ calculation window, the calculated interference terms of a vibration with any of the other vibrations have the same sign and add up to a positive contribution on the left and a negative contribution on the right of the spectrum. Further, the measured spec- trum becomes slightly dispersive around $1600 \mathrm{~cm}^{-1}$, indicating that the linear scan of the signal collection angle $\phi^{\prime}$ deviates from the direction in which the nonresonant background is a minimum.

On lowering the FAD concentration, the sample layer becomes more transparent $(|\Delta \alpha|$ decreases $)$ and the mismatching method achieves higher background suppression ratios under the same conditions (Fig. 1.0). This compensates in part for the decrease in resonant signal due to the lower sample concentration; the CARS spectrum of a $1.4 \times 10^{-3} \mathrm{~mol} \mathrm{dm}{ }^{-3}$ FAD solution $(0.15 / 0.25 / 0.15 \mathrm{~mm}$ configuration), measured under the same mismatching conditions as the spectrum of Fig. 8(a), is shown in Fig. 9. The spectrum differs from that for a concentration of $5.7 \times 10^{-3} \mathrm{~mol} \mathrm{dm}^{-3}$ mainly in the higher background. This confirms the fact that the background is additive and does not interfere with the spectrum.

\section{COMPARISON OF THE MISMATCHING, POLARIZATION CARS AND RESONANCE CARS METHODS APPLIED TO FAD}

The reduction in the vibration resonant signal, in the case of the polarization CARS spectra, is determined by 


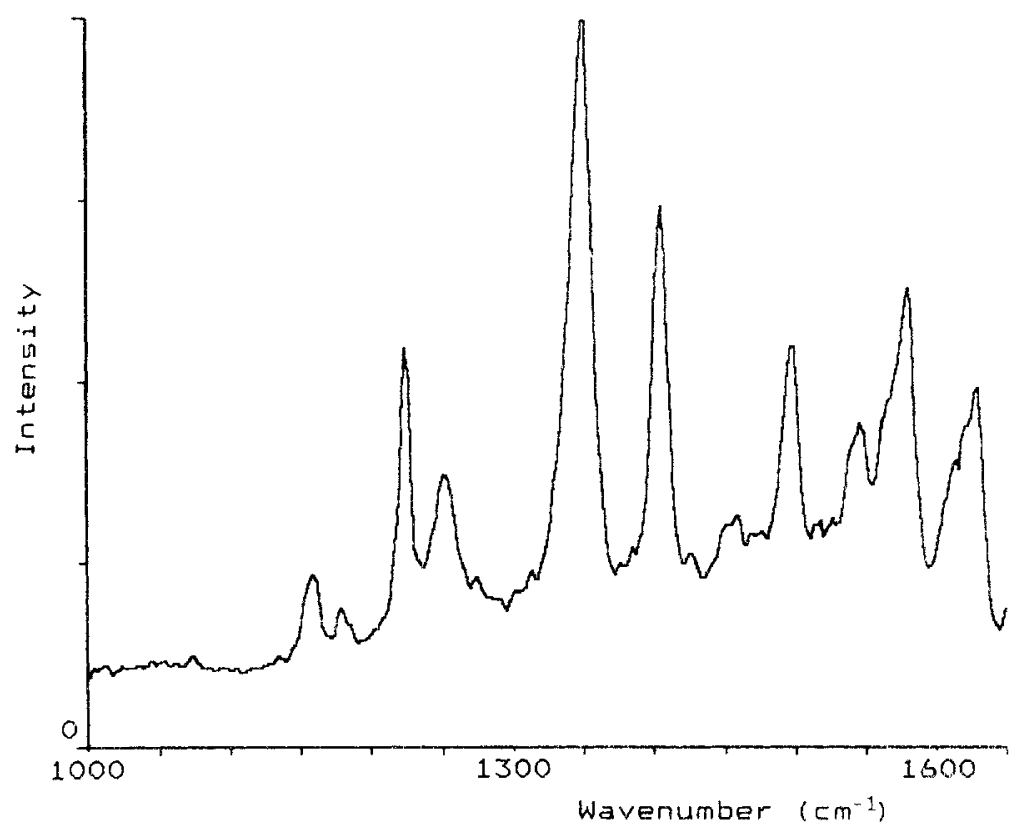

Figure 9. CARS spectrum (sum of five scans) of a $1.4 \times 10^{-3} \mathrm{~mol} \mathrm{dm}^{-3}$ FAD solution in a $0.15 / 0.25 / 0.15$ mm triple layer configuration, measured under the same conditions as in Fig. 8(a).

the difference in the depolarization ratios of the resonant and non-resonant susceptibilities. For the 1347 $\mathrm{cm}^{-1}$ vibration a loss factor of ca. 900 is measured. The signal loss in the case of the background suppression technique by phase mismatching is much lower and results from the partial mismatch inside the sample layer and the reduced collection angle aperture. For the $5.7 \times 10^{-3} \mathrm{~mol} \mathrm{dm}^{-3}$ concentration, the $1347 \mathrm{~cm}^{-1}$ peak intensity is a factor of 15-20 smaller than its contribution to the intensity in the matched spectrum. For the $1.4 \times 10^{-3} \mathrm{~mol} \mathrm{dm}^{-3}$ solution this factor is $25-35$.

For the pump and Stokes energy, photomultiplier setting and sample concentration that apply to the polarization CARS spectra, the $1347 \mathrm{~cm}^{-1}$ peak intensity of the spectra in Figs 8(a) and 9 is $50-100$ times larger than that in Fig. 4 [even disregarding the larger sample length $(0.9 \mathrm{~mm})$ used for the polarization CARS measurements]. In general, with a higher signal level, the pump and Stokes pulse energies can be lowered, reducing the possibility of photodegradation of the sample.

As stated before, the improvement achieved in the ratio of the resonant signal and non-resonant background is less than 10 for the polarization CARS spectra in Fig. 4. On reducing the sample concentration to $1.4 \times 10^{-3} \mathrm{~mol} \mathrm{dm}^{-3}$, this figure would decrease below 1 . The opposite is the case for the mismatching method: for the $5.7 \times 10^{-3} \mathrm{~mol} \mathrm{\textrm {dm } ^ { - 3 }}$ solution the improvement is ca. 20 , whereas for the more transparent $1.4 \times 10^{-3} \mathrm{~mol} \mathrm{dm}^{-3}$ sample the improvement is ca. 150. In fact, these figures must be divided by approximately three, because owing to the better glass/sample thickness ratio, the resonant-to-non-resonant intensity of the normal (matched) CARS spectrum was shown to be a factor of 3 higher for the configuration with a $0.9-\mathrm{mm}$ sample layer $\left(l_{\mathrm{s}} \gg l_{\mathrm{g}}\right)$, compared with a $0.25-\mathrm{mm}$ sample layer $\left(l_{\mathrm{s}} \approx l_{\mathrm{g}}\right)$.

The quality of the spectrum for the $1.4 \times 10^{-3} \mathrm{~mol}$ $\mathrm{dm}^{-3}$ solution (Fig. 9) is better than ${ }^{12}$ or comparable to $^{17}$ the resonance CARS spectra of flavins of the same concentration excited at $480 \mathrm{~nm}$. Both the mismatching technique and the resonance CARS technique give positive Lorentzian spectral bands, facilitating the determination of band positions and widths. It should be noted that the interference between the resonant susceptibilities of neighbouring vibrations is also present in resonance CARS spectra and may affect the band wavenumbers and intensities determined directly from the peak position and height.

For positive Lorentzian bands of flavins, resonance excitation using a two-laser CARS process (i.e. singly degenerate) is restricted to a pump wavelength of 480 $\mathrm{nm}{ }^{18}$ The mismatching method is not restricted in this way.

The resonance CARS technique, at a 480 -nm pump wavelength, yields much higher signal levels. From preliminary results, we can confirm the order of the pump and Stokes energies used by Dutta et al. ${ }^{12}(10-25 \mu \mathrm{J}$ per pulse) to obtain a significant CARS signal, which roughly indicates a $10^{2}-10^{3}$ times higher intensity level compared with the spectrum for the $1.4 \times 10^{-3} \mathrm{~mol}$ $\mathrm{dm}^{-3}$ FAD solution in Fig. 9.

The use of the phase-mismatching method under rigorous resonance conditions at a pump wavelength of $480 \mathrm{~nm}$ appears to be possible; the value of $\delta$ is then given by $\delta \approx-l_{s}\left(2 \alpha_{\mathrm{p}}-\alpha_{\mathrm{c}}\right) / 2$, which is -0.2 for a $1.4 \times 10^{-3} \mathrm{~mol} \mathrm{dm}^{-3} \mathrm{FAD}$ concentration in a $0.15 /$ $0.25 / 0.15 \mathrm{~mm}$ configuration, and is nearly constant over the range $1000-1650 \mathrm{~cm}^{-1}$. This situation cannot, according to the theory in the Appendix, be distinguished from a FAD solution excited at $532 \mathrm{~nm}$, for which $\delta$ is near 0.2 . This would correspond to an FAD concentration between $5.7 \times 10^{-3}$ and $1.4 \times 10^{-3} \mathrm{~mol}$ $\mathrm{dm}^{-3}$ for which the mismatching method is demonstrated. The sensitivity of the resonance CARS technique and the background suppression of the phase-mismatching method would thereby be combined. 
At ca. $488 \mathrm{~nm}$ the condition $\Delta \alpha=0$ is approximated (for a shift of $1300 \mathrm{~cm}^{-1}$ ) and an optimum background suppression is theoretically expected. It should be noted, however, that at high peak intensities and absorption rates of the pump and Stokes beams, a deviation from the exponential absorption mechanism may occur, owing to a substantial stimulated emission of absorbed radiation. The absorption at the pump and Stokes wavelengths then becomes linear in the sample layer thickness and the derivation given in the Appendix should accordingly be corrected. (This linearization indicates a saturation of the resonantly excited molecules and is, from a spectroscopic point of view, usually unwanted. According to unpublished measurements on Rhodamine $6 \mathrm{G}$ solutions excited at $532 \mathrm{~nm}$, a further increase in the pump energy merely enhances the non-saturated solvent background and decreases the signal-to-background ratio.)

Comparison of the band positions of FAD summarized in Table 1 and those summarized by Morris and Bienstock ${ }^{16}$ (disregarding a systematic difference of $4 \mathrm{~cm}^{-1}$ ) shows good agreement with the band positions determined by spontaneous Raman scattering of a $2 \times 10^{-3} \mathrm{~mol} \mathrm{dm}^{-3} \mathrm{KI}$-quenched FAD solution.

\section{CONCLUSIONS}

The polarization-sensitive CARS spectra of FAD, preresonantly excited at $532 \mathrm{~nm}$, show 15 bands in the range $1000-1650 \mathrm{~cm}^{-1}$. The depolarization ratios of these bands are seen to be close to the depolarization ratio of the non-resonant background. As a result, the intensity of the analysed signal is low and the improvement in the ratio of the resonant signal and non-resonant background is poor $(\leqslant 10)$. The depolarization ratios, however, can be estimated accurately from a set of polarization CARS spectra recorded with small offsets in the analyser orientation. This also easily reveals the presence of weak vibrations.

A background suppression technique using coherent cancellation of non-resonant signals through phase mis- matching has been demonstrated for (pre)resonantly excited FAD. Spectra with positive Lorentzian bands are obtained for $5.7 \times 10^{-3}$ and $1.4 \times 10^{-3} \mathrm{~mol} \mathrm{dm}^{-3}$ FAD solutions. The signal level is $50-100$ times higher than in the polarization CARS measurement, and an improvement in the resonant signal-to-background ratio of ca. 50 is achieved for the lowest concentration. The remaining non-resonant background is additive, and does not interfere with the vibration resonant signals. The quality of the spectra is comparable to that of previously published spectra recorded under rigorous resonance conditions.

From the spectra obtained with the mismatching technique, the band position and bandwidth can almost directly be determined. For the peak intensity, especially for weak vibrations, interference between neighbouring vibrations must be accounted for.

It is expected that the background suppression technique by phase mismatching will be applicable to a variety of (pre)resonantly excited solutions, of which FAD is only an example. However, depending on the sample absorption, the non-resonant susceptibility ratio (the real part, $F_{\mathrm{r}}$ ) of cuvette windows and sample and the layer thicknesses, the application of the mismatching technique (i.e. finding a good minimum in the non-resonant background) may not always be as straightforward as with FAD (as is indicated, for example, by the bottom row in Fig. 10).

We have not yet tried to apply the mismatching technique under rigorous resonance conditions, or the special situation in which $\Delta \alpha=0$. It would be interesting to investigate the theoretically predicted dependence of the non-resonant intensity on the parameters of Eqn (A10) as shown in Fig. 10.

\section{Acknowledgements}

The financial support of the KSLA (Royal Dutch Shell Laboratory, Amsterdam) and the STW (Foundation for Technical Sciences), which made this work possible, is gratefully acknowledged.

\section{REFERENCES}

1. F. W. Schneider, in Non-Linear Raman Spectroscopy and its Chemical Applications, edited by W. Kiefer and D. A. Long, p. 445. Reidel, Dordrecht (1982).

2. S. A. J. Druet and J.-P. E. Taran, Prog. Quantum Electron. 7, 1 (1981)

3. W. M. Tolles, J. W. Nibler, J. R. McDonald and A. B. Harvey, Appl. Spectrosc. 31, 253 (1977).

4. S. A. Akhmanov and N. I. Koroteev, Sov. Phys. Usp. 20, 899 (1977).

5. I. Chabay, G. K. Klauminzer and B. S. Hudson, Appl. Phys. Lett. 28, 27 (1976)

6. R. T. Lynch, S. D. Kramer, H. Lotem and N. Bloembergen, Opt. Commun. 16, 372 (1976).

7. F. M. Kamga and M. G. Skeats, Opt. Lett. 5. 126 (1980).

8. R. Brakel, V. Mudogo and F. W. Schneider, J. Chem. Phys. 84, 2451 (1986).

9. T. A. H. M. Scholten, G. W. Lucassen, F. F. M. de Mul and J. Greve, Appl. Opt. in press.

10. Y. Yacoby, R. Fitzgibbon and B. Lax, J. Appl. Phys. 51, 3072 (1980).
11. J. Behringer, in Raman Spectroscopy, edited by H. A. Szymanski, p. 205. Plenum Press, New York (1967).

12. P. K. Dutta, J. R. Nestor and T. G. Spiro, Proc. Natl. Acad. Sci USA 74, 4146 (1977).

13. G. L. Eesley, Coherent Raman Spectroscopy. Pergamon Press, New York (1981)

14. T. A. H. M. Scholten, G. W. Lucassen, F. F. M. de Mul and J. Greve, Appl. Opt. 27, 3225 (1988)

15. Biochemical Tables, BHD Chemicals, England

16. M. D. Morris and R. J. Bienstock, in Spectroscopy of Biological Systems, edited by R. J. H. Clark and R. E. Hester. Wiley, Chichester (1986).

17. A. J. W. G. Visser, J. Vervoort, D. J. O'Kane and L. A. Carreira, Eur. J. Biochem. 131, 639 (1983)

18. P. K. Dutta and T. G. Spiro, J. Chem. Phys. 69, 3119 (1978).

19. J. W. Fleming and C. S. Johnson, Jr, J. Raman Spectrosc. 8, 284 (1979).

20. J. R. Andrews, R. M. Hochstrasser and H. P. Trommsdorff, Chem. Phys. 62, 87 (1981). 


\section{APPENDIX. THEORY OF NON-RESONANT BACKGROUND SUPPRESSION BY PHASE MISMATCHING}

In this Appendix, the intensity equation for the general situation of a triple layer with an absorbing intermediate sample layer is derived. The triple layer configuration depicted in Fig. 1 will be considered. It is assumed that the electric fields are plane, monochromatic waves, travelling in the $z$-direction. The fields are attenuated in an exponential way, while propagating through the sample layer. The pump and Stokes fields $\left(\mathbf{E}_{\mathrm{p}}, \mathbf{E}_{\mathrm{s}}\right)$ are then given by (disregarding reflection losses at the layer boundaries and assuming $z=0$ at the leftmost boundary)

$$
\begin{aligned}
& \text { layer } 1: \mathbf{E}_{\mathrm{p}, \mathrm{s}_{1}}=\mathbf{e}_{\mathrm{p}, \mathrm{s}} E_{\mathrm{p}, \mathrm{s}_{0}} \\
& \quad \times \exp \left[-i\left(\omega_{\mathrm{p}, \mathrm{s}} t-k_{\mathrm{p}, \mathrm{s} 1} z\right)\right] \\
& \text { layer } 2: \mathbf{E}_{\mathbf{p}, \mathrm{s}_{2}}=\mathbf{e}_{\mathrm{p}, \mathrm{s}} E_{\mathrm{p}, \mathrm{s} 0} \\
& \quad \times \exp \left[-i\left(\omega_{\mathrm{p}, \mathrm{s}} t-k_{\mathrm{p}, \mathrm{s} 2} z-\theta_{\mathbf{p}, \mathrm{s}_{12}}\right)\right. \\
& \left.\quad-\frac{1}{2} \alpha_{\mathrm{p}, \mathrm{s}}\left(z-l_{\mathrm{g}}\right)\right] \\
& \text { layer } 3: \mathbf{E}_{\mathrm{p}, \mathrm{s} 3}=\mathbf{e}_{\mathrm{p}, \mathrm{s}} E_{\mathrm{p}, \mathrm{s} 0} \\
& \quad \times \exp \left[-i\left(\omega_{\mathrm{p}, \mathrm{s}} t-k_{\mathrm{p}, \mathrm{s} 3} z-\theta_{\mathrm{p}, \mathrm{s} 12}-\theta_{\mathrm{p}, \mathrm{s} 23}\right)\right. \\
& \left.\quad-\frac{1}{2} \alpha_{\mathrm{p}, \mathrm{s}} l_{\mathrm{s}}\right]
\end{aligned}
$$

with

$$
\begin{aligned}
\theta_{\mathrm{p}, \mathrm{s}_{12}}=\left(k_{\mathrm{p}, \mathrm{s}_{1}}-k_{\mathrm{p}, \mathrm{s}_{2}}\right) l_{\mathrm{g}} & ; \\
\theta_{\mathrm{p}, \mathrm{s}_{23}} & =\left(k_{\mathrm{p}, \mathrm{s}_{2}}-k_{\mathrm{p}, \mathrm{s}_{3}}\right)\left(l_{\mathrm{s}}+l_{\mathrm{g}}\right)
\end{aligned}
$$

where $e_{p}$ and $e_{s}$ denote the polarization directions of the linearly polarized pump and Stokes fields, respectively; $\alpha_{p}$ and $\alpha_{s}$ are the (intensity) absorption coefficients of the sample layer at the pump and Stokes frequencies $\omega_{p}$ and $\omega_{\mathrm{s}}$ (the subscripts $\mathrm{p}$ and $\mathrm{s}$ denote the pump or Stokes beams; the numbers $0,1,2,3$ denote free space, layer 1, layer 2 and layer 3 , respectively).

The third-order polarization at the CARS frequency $\omega_{\mathrm{c}}=2 \omega_{\mathrm{p}}-\omega_{\mathrm{s}}$ can now be calculated for each of the layers $i$, according to

$$
\mathbf{P}_{\mathbf{i}}^{(3)} \propto \boldsymbol{\chi}_{\mathbf{i}}^{(3)} \cdot \mathbf{E}_{\mathbf{p i}_{\mathrm{i}}}^{2} \cdot \mathbf{E}_{\mathbf{s i}_{\mathbf{i}}}^{*}
$$

The signal field at a point $z_{0}$ on the $z$-axis (outside the triple layer) consists of the sum of the fields induced by every point of the polarized medium and can be written as

$$
\mathbf{E}_{\mathrm{c}}\left(z_{0}\right) \propto \int_{z^{\prime}} \mathbf{P}^{(3)}\left(z^{\prime}\right) \cdot G\left(z_{0}, z^{\prime}\right) \mathrm{d} z^{\prime}
$$

where the subscript c denotes CARS. The function $G$ accounts for the optical pathlength and the absorption of the signal field between the origin $z^{\prime}$ of the wavelet $\mathbf{P}^{(3)}\left(z^{\prime}\right)$, and the position $z_{0}$. For the different layers it is given by

$$
\left.\begin{array}{r}
\text { (1) } \exp \left[i \left(k_{\mathrm{c}_{1}}\left[l_{\mathrm{g}}-z^{\prime}\right]+k_{\mathrm{c}_{2}} l_{\mathrm{s}}+k_{\mathrm{c}_{3}} l_{\mathrm{g}}\right.\right. \\
\left.\left.+k_{\mathrm{c}_{0}}\left[z_{0}-\left\{2 l_{\mathrm{g}}+l_{\mathrm{s}}\right\}\right]\right)-\frac{1}{2} \alpha_{\mathrm{c}} l_{\mathrm{s}}\right] \\
\text { (2) } \exp \left[i \left(\mathbf{k}_{\mathrm{c}_{2}}\left[l_{\mathrm{g}}+l_{\mathrm{s}}-z^{\prime}\right]+k_{\mathrm{c}_{2}} l_{\mathrm{g}}\right.\right. \\
\left.\left.\left.+k_{\mathrm{c}_{0}}\left[z_{0}-\left\{2 l_{\mathrm{g}}+l_{\mathrm{s}}\right\}\right]\right)-\frac{1}{2} \alpha_{\mathrm{c}} l_{\mathrm{g}}+l_{\mathrm{s}}-z^{\prime}\right)\right] \\
\text { (3) } \exp \left[i \left(k_{\mathrm{c}_{3}}\left[\left\{2 l_{\mathrm{g}}+l_{\mathrm{s}}\right\}-z^{\prime}\right]\right.\right. \\
\left.\left.+k_{\mathrm{c}_{0}}\left[z_{0}-\left\{2 l_{\mathrm{g}}+l_{\mathrm{s}}\right\}\right]\right)\right]
\end{array}\right\}
$$

The signal field is then given by

$$
\begin{aligned}
\mathbf{E}_{\mathrm{c}} \propto \exp \left(-\frac{1}{2} \alpha_{\mathrm{c}} l_{\mathrm{s}}\right)\left\{\chi_{\text {window }}^{\mathrm{NR}} \frac{\sin \left(\phi_{1}\right)}{\Delta k_{1}}\right. \\
\times\left(\exp \left[2 i\left(\phi_{1}+\phi_{2}\right)-\Delta \alpha l_{\mathrm{s}}\right]+1\right)+\chi_{\text {sample }} \exp \left(i \phi_{1}\right) \\
\left.\quad \times \frac{\left[\exp \left(2 i \phi_{2}-\Delta \alpha l_{\mathrm{s}}\right)-1\right]}{2\left(-\Delta \alpha+i \Delta k_{2}\right)}\right\} \mathbf{e}_{\mathrm{p}} \cdot \mathbf{e}_{\mathrm{p}} \cdot \mathbf{e}_{\mathrm{s}} \quad \text { (A6) }
\end{aligned}
$$

where $\Delta \alpha=\frac{1}{2}\left(2 \alpha_{\mathrm{p}}+\alpha_{\mathrm{s}}-\alpha_{\mathrm{c}}\right), \Delta k_{1}=2 k_{\mathrm{p}_{1}}-k_{\mathrm{s}_{1}}-k_{\mathrm{c}_{1}}$ for layers 1 and $3, \Delta k_{2}=2 k_{\mathrm{p}_{2}}-k_{\mathrm{s}_{2}}-k_{\mathrm{c}_{2}}$ for layer $2, \phi_{1}=$ $\Delta k_{1} l_{\mathrm{g}} / 2$ and $\phi_{2}=\Delta k_{2} l_{\mathrm{s}} / 2$.

Since the suppression of the non-resonant intensity is of major concern, the vibration resonant contribution of the sample layer will not be considered here. It is assumed that the polarization orientation of the nonresonant contributions of the window and sample are the same (this is the case for transparent media where the polarization orientation is determined by Kleinman symmetry; otherwise it can be achieved by analysing the signal with a polarizer). The non-resonant susceptibility of the sample layer can then be expressed in the nonresonant susceptibility of the window material:

$$
\begin{aligned}
\mathbf{e}_{\mathrm{c}} \cdot \boldsymbol{\chi}_{\mathrm{sample}}^{\mathrm{NR}} \cdot \mathbf{e}_{\mathrm{p}} \cdot \mathbf{e}_{\mathrm{p}} \cdot \mathbf{e}_{\mathrm{s}} \\
=\left(F_{\mathrm{r}}+i F_{\mathrm{i}}\right) \mathbf{e}_{\mathrm{c}} \cdot \boldsymbol{\chi}_{\text {window }}^{\mathrm{NR}} \cdot \mathbf{e}_{\mathrm{p}} \cdot \mathbf{e}_{\mathrm{p}} \cdot \mathbf{e}_{\mathrm{s}}
\end{aligned}
$$

where $e_{c}$ is the unit vector giving the polarization orientation of signal field. $F_{\mathrm{r}}$ and $F_{\mathrm{i}}$ are real and can be regarded as constant over the frequency range of interest.

The non-resonant CARS intensity is then given by $\left|\mathbf{e}_{\mathrm{c}} \cdot \mathbf{E}_{\mathrm{c}}\right|^{2}$ :

$$
\begin{aligned}
I_{\mathrm{NR}} \propto & l_{\mathrm{s}}^{2}\left\{( \frac { l _ { \mathrm { g } } } { l _ { \mathrm { s } } } ) ^ { 2 } \operatorname { s i n c } ^ { 2 } ( \phi _ { 1 } ) \left[\left(\mathrm{e}^{\delta}+1\right)^{2} \cos ^{2}\left(\phi_{1}+\phi_{2}\right)\right.\right. \\
& \left.+\left(\mathrm{e}^{\delta}-1\right)^{2} \sin ^{2}\left(\phi_{1}+\phi_{2}\right)\right]+\left(F_{\mathrm{r}}^{2}+F_{\mathrm{i}}^{2}\right) \\
& \times \frac{\left(\mathrm{e}^{\delta}-1\right)^{2} \cos ^{2}\left(\phi_{2}\right)+\left(\mathrm{e}^{\delta}+1\right)^{2} \sin ^{2}\left(\phi_{2}\right)}{4 \phi_{2}{ }^{2}+\delta^{2}} \\
+ & 2\left(\frac{l_{\mathrm{g}}}{l_{\mathrm{s}}}\right) \operatorname{sinc}\left(\phi_{1}\right) \\
\times & \left\langle X\left(\mathrm{e}^{2 \delta}-1\right) \cos \left(\phi_{1}\right)-Y\left[\left(\mathrm{e}^{\delta}-1\right)^{2}\right.\right. \\
\times & \left.\frac{\left.\left.\sin \left(\phi_{1}\right)-4 \mathrm{e}^{\delta} \sin \left(\phi_{2}\right) \cos \left(\phi_{1}+\phi_{2}\right)\right]\right\rangle}{4 \phi_{2}^{2}+\delta^{2}}\right\}
\end{aligned}
$$

where $X=\delta F_{\mathrm{r}}+2 \phi_{2} F_{i}, Y=-\delta F_{i}+2 \phi_{2} F_{\mathrm{r}}$ and $\delta=$ $-\Delta \alpha l_{s}$.

The second term of Eqn (A8) represents the nonresonant contribution originating from the sample layer; disregarding the factor $F_{\mathrm{r}}{ }^{2}+F_{\mathrm{i}}{ }^{2}$, it gives the phase-match factor of an absorbing sample layer. ${ }^{20}$ Since the first and second terms are positive, a reduction in the background $I_{\mathrm{NR}}$ must result from a negative contribution of the third factor of Eqn (A8).

A special situation occurs for $\Delta \alpha=0$, which not only represents the case of a transparent sample layer $\left(\alpha_{\mathrm{p}, \mathrm{s}, \mathrm{c}}=0\right)$ but also the situation in which the position of the pump, Stokes and CARS wavelength within the 
absorption band of the excited molecule is such that $\alpha_{c}=2 \alpha_{p}+\alpha_{s}$; Eqn (A8) then reduces to

$I_{\mathrm{NR}}(\Delta \alpha=0) \propto l_{\mathrm{s}}^{2} \operatorname{sinc}^{2}\left(\phi_{2}\right)$

$$
\times\left\{F_{\mathrm{i}}^{2}+\left[F_{\mathrm{r}}+2\left(\frac{l_{\mathrm{g}}}{l_{\mathrm{s}}}\right) \frac{\operatorname{sinc}\left(\phi_{1}\right)}{\operatorname{sinc}\left(\phi_{2}\right)} \cos \left(\phi_{1}+\phi_{2}\right)\right]^{2}\right\}
$$

The second term within the braces can theoretically be reduced to zero by phase mismatching. ${ }^{9}$ The imaginary part of the non-resonant background, however, cannot be compensated. Thus, for the condition $\Delta \alpha=0$, the non-resonant contribution of the solvent can be compensated and the obtained spectrum of the resonantly excited molecule includes interferences of the imaginary part of the (its own) non-resonant background.

With this in mind, Eqn (A8) can be further simplified by regarding only a possible suppression of the real part $F_{\mathrm{r}}$; it then reduces to a second-order polynomial in $F_{\mathrm{r}}$. In this case, the non-resonant intensity of the triple layer, normalized by the non-resonant intensity which

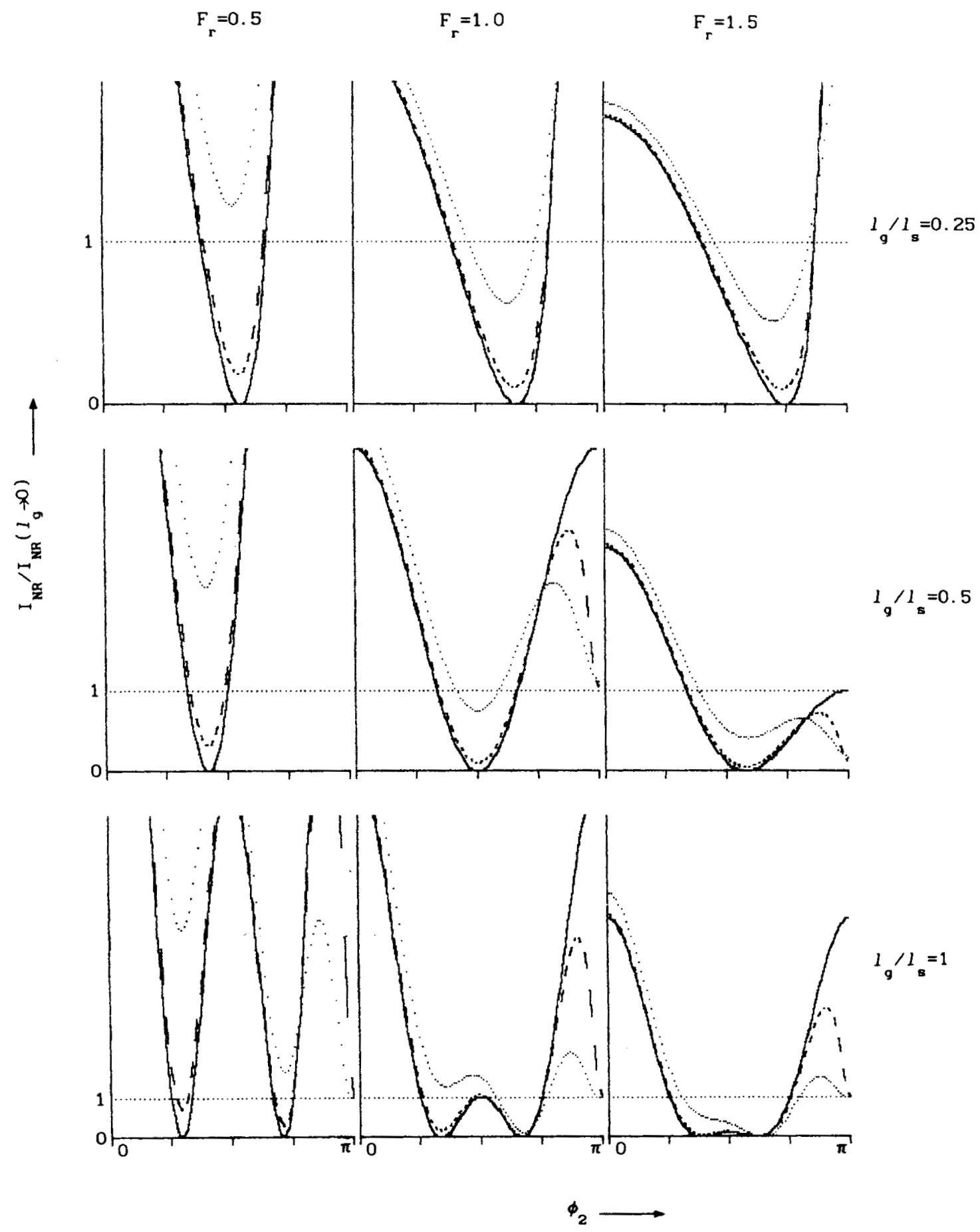

Figure 10. Plots of the calculated ratio $I_{\mathrm{NR}} / I_{\mathrm{NR}}\left(I_{\mathrm{a}} \rightarrow 0\right.$ ) [Eqn (A10)] as a function of $\phi_{2}=\Delta k_{2} / \mathrm{g} / 2$ (ranging from 0 to $\pi$ ). Solid curves, $\delta=0$ (no absorption of the sample layer, or $2 \alpha_{0}+\alpha_{s}-\alpha_{c}=0$ ); dashed curves, $\delta= \pm 0.4$; dotted curves, $\delta= \pm 1.1$. The horizontal dotted line indicates the value 1 . From left to right the value of $F_{r}$ is $0.5,1$ and 1.5 ; from top to bottom the window thickness $l_{g}$ is $0.25,0.5$ and 1 times the sample layer thickness $l_{\mathrm{s}}$. The mismatches $\Delta k_{1}$ and $\Delta k_{2}$ are assumed to be equal. 
would result from the sample layer alone [the second term of Eqn (A8)], is then proportional to

$$
\begin{aligned}
\frac{I_{\mathrm{NR}}}{I_{\mathrm{NR}}\left(l_{\mathrm{g}} \rightarrow 0\right)} & \propto 1+\left(\frac{l_{\mathrm{g}}}{l_{\mathrm{s}}}\right) \frac{\operatorname{sinc}\left(\phi_{1}\right)}{F_{\mathrm{r}}} \frac{1}{P} \\
\times & {\left[\left(4 \phi_{2}{ }^{2}+\delta^{2}\right)\left(\frac{l_{\mathrm{g}}}{l_{\mathrm{s}}}\right) \frac{\operatorname{sinc}\left(\phi_{1}\right)}{F_{\mathrm{r}}} Q+2 R\right] }
\end{aligned}
$$

where

$$
\begin{aligned}
P= & {\left[\mathrm{e}^{\delta}-1\right]^{2} \cos ^{2}\left(\phi_{2}\right)+\left[\mathrm{e}^{\delta}+1\right]^{2} \sin ^{2}\left(\phi_{2}\right) } \\
Q= & {\left[\mathrm{e}^{\delta}+1\right]^{2} \cos ^{2}\left(\phi_{1}+\phi_{2}\right)+\left[\mathrm{e}^{\delta}-1\right]^{2} \sin ^{2}\left(\phi_{1}+\phi_{2}\right) } \\
R= & \delta\left(\mathrm{e}^{2 \delta}-1\right) \cos \left(\phi_{1}\right)-2 \phi_{2}\left[\left(\mathrm{e}^{\delta}-1\right)^{2} \sin \left(\phi_{1}\right)\right. \\
& \left.-4 \mathrm{e}^{\delta} \sin \left(\phi_{2}\right) \cos \left(\phi_{1}+\phi_{2}\right)\right]
\end{aligned}
$$

Since the vibration resonant signal of the sample layer is governed by the same phase-match factor as the non-resonant background $I_{\mathrm{NR}}\left(l_{\mathrm{g}} \rightarrow 0\right)$, the reciprocal of Eqn (A10) gives the improvement in the ratio of the vibration resonant signal and the non-resonant background, compared with the same ratio in the absence of the cell windows. Equation (A10) can best be evaluated by numerical analysis. It is found that this equation is symmetrical in $\delta$.

In Fig. 10 some calculations are shown; each plot gives the value of Eqn (A10) as a function of $\phi_{2}=$ $\Delta k_{2} l_{\mathrm{s}} / 2$, varied from 0 to $\pi$. In the experimental situation $\phi_{2}$ is varied by changing $\Delta k_{2}$, which is determined by the beam crossing angle $\theta$ [see Eqn (2)], rather than $l_{s}$. Three curves are shown in each plot, corresponding to an absorption factor $\exp (\delta)$ equal to 1 (no absorption, solid line), 1.5 (dashed line) and 3 (dotted line). Note that the curves with $\exp (\delta)=1.5$ and 3 also cover the absorptions $\exp (-\delta)=0.67$ and 0.33 , respectively. From the left to the right the value of $F_{\mathrm{r}}$ is $0.5,1$ and 1.5 [values of $F_{\mathrm{r}}$ for transparent media (with respect to the non-resonant susceptibility of glass) are in this range; for quartz, water and ethanol $F_{\mathrm{r}}$ was measured to be $0.63,0.88$ and 1.25 , respectively]. From the top to the bottom, the thickness $l_{\mathrm{g}}$ of the cell windows is varied: $0.25 l_{\mathrm{s}}, 0.5 l_{\mathrm{s}}$ and $l_{\mathrm{s}}$. It is assumed, in these plots, that the dispersion of the cell windows and sample layer and thus $\Delta k_{1}$ and $\Delta k_{2}$ are equal.

For the dotted, horizontal line in the plots, the nonresonant background of the total triple layer equals the non-resonant background of the sole sample layer (under the same conditions). In this case, the value of Eqn (A10) is 1 and the non-resonant contribution from the cell windows is in fact compensated. For points below this line an improvement in the signal-to-background ratio in the spectrum of the sample occurs. The improvement, however, is at the cost of the vibration resonant signal strength, which depends on the mismatch $\phi_{2}(=$ position on the horizontal axis) and is given by the above-mentioned phase-match factor of the sample layer. For transparent samples $[\exp (\delta)=1]$ this decrease is given by the well known $\operatorname{sinc}^{2}\left(\phi_{2}\right)$ function.

It is seen from Fig. 10 that for a transparent sample layer the non-resonant background can be reduced to zero in all cases. From the point of signal loss, a relatively thick cell window is favourable.

For an absorbing sample layer, with $\exp (\delta)$ smaller or larger than 1, background reduction is not possible under all circumstances. For values of $F_{\mathrm{r}}$ smaller than 1, the thickness $l_{\mathrm{g}}$ can be made small to obtain a better (if any) suppression of the non-resonant background. However, this is at the cost of the signal intensity. For values of $F_{\mathrm{r}}$ larger than 1 , the suppression may be improved by increasing the thickness $l_{\mathrm{g}}$ of the cell windows; in the lower right plot all curves reach a value below $2 \times 10^{-3}$. In general, a better suppression of the non-resonant background is achieved for $\delta$ closer to zero. 